\title{
A Convenient synthesis of 1-(diethoxyphosphoryl)cyclopropanecarboxylates
}

\author{
Katarzyna Wąsek ${ }^{1}$, Jacek Kędzia, ${ }^{1}$ Henryk Krawczyk ${ }^{1}$ \\ Jakub Wojciechowski ${ }^{2}$ and Wojciech M. Wolf ${ }^{2}$ \\ ${ }^{1}$ Institute of Organic Chemistry and ${ }^{2}$ Institute of General and Ecological Chemistry, Technical \\ University of Łódź, Żeromskiego 116, 90-924 Łódź, Poland \\ E-mail: henkrawc@p.lodz.pl
}

\begin{abstract}
An efficient synthesis of a series of 1-(diethoxyphosphoryl)cyclopropancarboxylates was accomplished by the reaction of terminal 1,2-diols cyclic sulfates with triethylphosphonoacetate. The stereochemistry of 2-benzyloxymethyl-1-(diethoxyphosphoryl)cyclopropanecarboxylic acid was determined by the single crystal X-ray structure analysis.
\end{abstract}

Keywords: Cyclopropanation, cyclopropanecarboxylates, 2,2-dioxo-1,3,2-dioxathiolanes, 1(diethoxyphosphoryl)cyclopropanecarboxylates, X-ray analysis

\section{Introduction}

During the last several years cyclopropanation of dialkyl malonates with 4-alkyl-2,2-dioxo-1,3,2dioxathiolanes has emerged as a versatile method for construction of cyclopropane carboxylic acid derivatives - the attractive synthons of aminocyclopropanecarboxylic acids and various carbo- and heterocyclic coumpounds. ${ }^{1-8}$ It is well documented that succeeding steps of this reaction consist in fully regioselective dioxathiolane ring opening with the malonate anion and intramolecular $\mathrm{S}_{\mathrm{N}} 2$ type substitution of a sulfate residue in the resulting intermediate. Surprisingly, similar cyclopropanation of acetates activated by other electron withdrawing groups remains almost unexplored. To the best our knowledge the literature contains only one example of such a reaction. It has been demonstrated that the base promoted cyclopropanation of $t$-butyl dimethoxyphosphorylacetate with (S)-4-methyl-2,2-dioxo-1,3,2-dioxathiolane proceeds highly regio- and stereoselective, affording $t$-butyl trans-(1R,2R)-1-dimethoxyphosphoryl-2methylcyclopropanecarboxylate with ee $>99 \%$ and $d e 94 \%{ }^{9}$ 


\section{Results and Discussion}

In the search of an effective approach to C-2 functionalized trans-1aminocyclopropanephosphonic acids as potential biologically active compounds, we envisaged that the corresponding trans-2-alkyl-1-(dialkoxyphosphoryl)cyclopropanecarboxylates 4 might be their useful precursors.

In this paper, we demonstrate that the cyclopropanation reaction of alkyl dialkoxyphosphorylacetates with 2,2-dioxo-1,3,2-dioxathiolanes $\mathbf{2}$ has general applicability and that it can serve successfully as a source of different carboxylates 4 . We selected commercial triethylphosphonoacetate $\mathbf{3}$ as a model substrate and structurally various 2,2-dioxo-1,3,2dioxathiolanes $\mathbf{2 a - d}$ as representative cyclopropanating reagents. The thiolanes $\mathbf{2 a - d}$ were readily synthesized from the appropriate terminal 1,2-diols 1a-d following the routine one pot Sharpless procedure. ${ }^{1}$ The acylation of diols $\mathbf{1 a - d}$ with thionyl chloride followed by oxidation, with sodium periodinate in the presence of catalytic ruthenium chloride, afforded $\mathbf{2 a - d}$ in high yields (Scheme 1). The crude thiolanes $\mathbf{2 a - d}$ did not required purification to be used in subsequent reaction step.

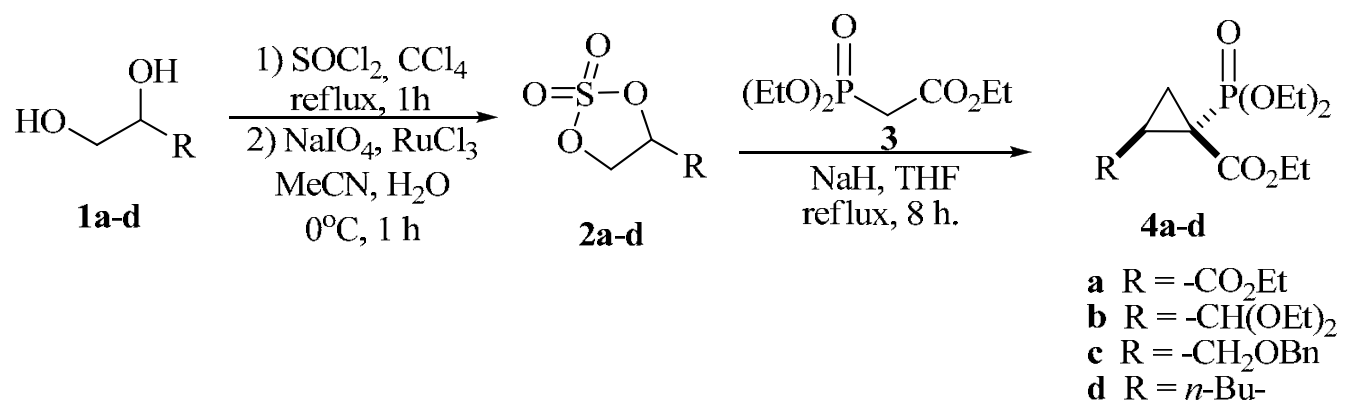

\section{Scheme 1}

Treatment of the thiolanes $\mathbf{2 a - d}$ with triethylphosphonoacetate $\mathbf{3}$ in the presence of two equivalents of $\mathrm{NaH}$ in $\mathrm{THF}$ at reflux for $8 \mathrm{~h}$ provided the corresponding cyclopropanes 4 a-d as single diastereoisomers in all cases (Scheme 1). Spectroscopic studies were not sufficient in determining the stereochemistry of the cyclopropanecarboxylates $\mathbf{4 a - d}$. The single crystal X-ray structure analysis of the 2-benzyloxymethyl-1-(diethoxyphosphoryl)-cyclopropanecarboxylic acid 5, which was prepared by base promoted hydrolysis of the cyclopropanecarboxylate 4c, (Scheme 2) showed that the phosphoryl and benzyloxymethyl groups are in mutual trans relationship (Figure 1). The cyclopropane endocyclic C-C bonds show a characteristic bondlength asymmetry which follows from the interactions of ring orbitals with $\pi$ system of a substituent. $^{14,15}$ The shortest bond (C2-C3) is located opposite the diethoxyphosphoryl and carboxylate substituents while the longest $(\mathrm{C} 1-\mathrm{C} 2)$ is placed in front of the unsubstituted endocyclic $\mathrm{C} 3$ atom. The $\mathrm{C} 1-\mathrm{C} 3$ is a distal bond for the benzyloxylmethyl substituent. In the crystal, molecules are linked by strong hydrogen bonds involving the phosphoryl O2 and carboxylate $\mathrm{O} 3$ atoms $\left[\mathrm{O} 2-\mathrm{H} 2\right.$ 0.85(6), O2 ${ }^{\cdots} \mathrm{O} 3 * 2.634(4), \mathrm{H} 2 \cdots \mathrm{O} 3 * 1.80(6)(\AA), \mathrm{O} 2-\mathrm{H} 2 \cdots \mathrm{O} 3 *$ 
170(3) $\left({ }^{\circ}\right)$; atom indicated with an asterisk is related by the $(x, y-1, z)$ symmetry operator] resulting in a ladder type arrangement extending along the [010] crystallographic axis. The crystal packing is additionally stabilized by the $\mathrm{C}-\mathrm{H}^{\cdots}{ }^{\cdots} \pi$ interactions ${ }^{16}$ between the phenyl rings and hydrogen atoms of the methylene groups. Therefore, taking into account the method of preparation and structural similarity, we by analogy assigned the trans configuration to the obtained products 4a-d. In this context, it is also worth nothing that the values of coupling constant ${ }^{3} J_{\mathrm{PH}}=16.0 \mathrm{~Hz}$ and ${ }^{3} J_{\mathrm{PH}}=16.5 \mathrm{~Hz}$ observed in ${ }^{1} \mathrm{H}$ NMR spectra of $4 \mathbf{a}$ and $\mathbf{5}$ respectively, were consistent with the synperiplanar arrangement of the phosphorus and H-2 atoms.

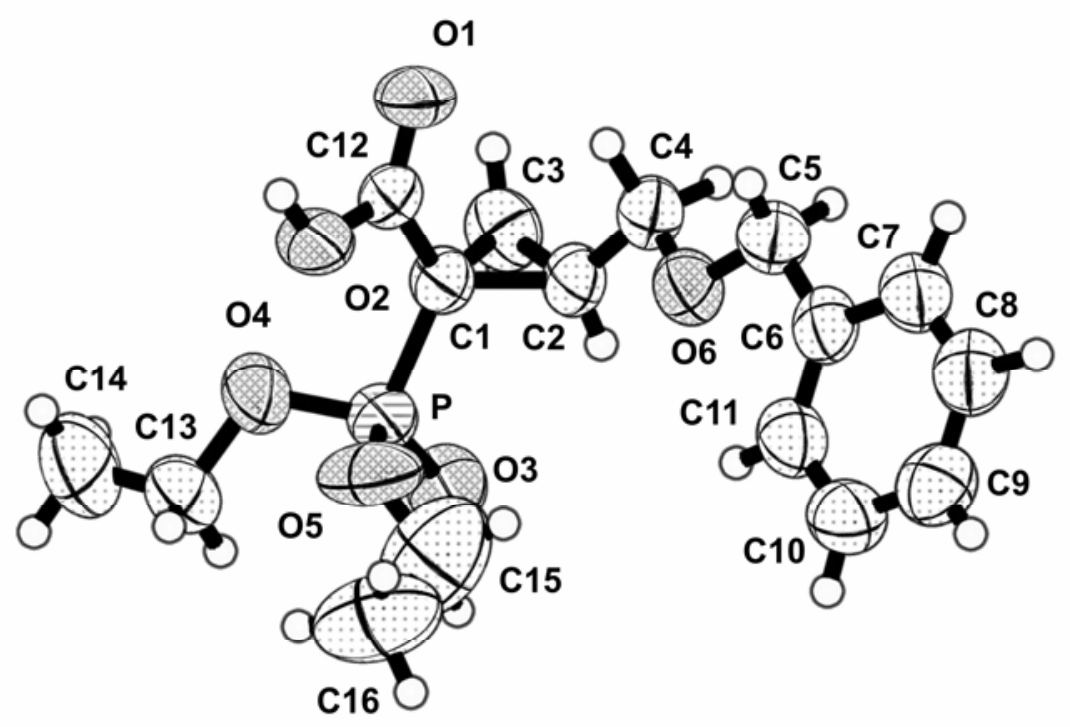

Figure 1. The molecular structure and numbering scheme of the cyclopropane carboxylic acid 5. Displacement ellipsoids are drawn at the $50 \%$ probability level. Hydrogen atoms are represented by circles of an arbitrary radius. Selected bond lengths $[\AA]$ : P-O3 1.466(2); P-C1 1.787(3); O1C12 1.197(3); O2-C12 1.312(4); O6-C4 1.417(4); C1-C3 1.521(4); C1-C2 1.554(3); C2-C3 1.472(5); C1-C12 1.492(4); C2-C4 1.493(4). Selected valency angles [ $\left.{ }^{\circ}\right]$ : P-C1-C2 112.6(2); PC1-C3 116.1(2); P-C1-C12 121.5(2); C1-C2-C3 60.3(2); C1-C3-C2 62.5(2); C2-C1-C3 57.2(2); C1-C2-C4 122.9(2); C3-C2-C4 123.9(3); C2-C1-C12 117.7(2) C3-C1-C12 115.2(2);. Selected torsion angles [ ${ }^{\circ}$ ]: P-C1-C2-C3 107.4(2); P-C1-C2-C4 -139.3(3); P-C1-C12-O2 13.9(3); O1C12-C1-C3 -17.7(4); O3-P-C1-C2 -32.7(2) ; O3-P-C1-C3 30.6(3); O3-P-C2-C12 179.6(2); O6C4-C3-C1 170.3(3); C4-C3-C2-C12 9.8(4). 


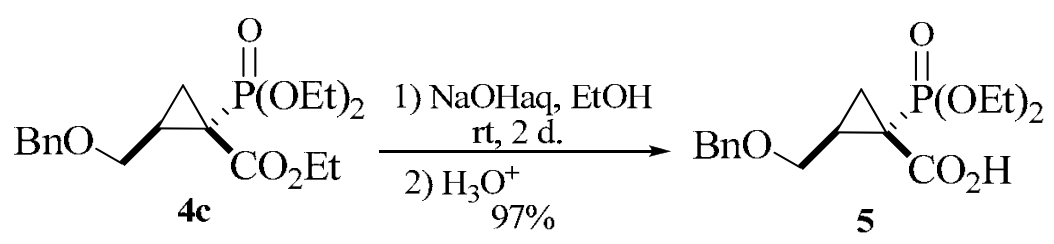

\section{Scheme 2}

\section{Conclusions}

In summary, our studies have clearly demonstrated general applicability of synthetic strategy based on cyclopropanation of triethylphosphonoacetate 3 with cyclic sulfates $\mathbf{2}$ of terminal 1,2diols for diastereoselective synthesis of substituted 1-(diethoxyphosphoryl) cyclopropanecarboxylates.

\section{Experimental Section}

General. Reagents were purchased from commercial sources and used as received without purification. Solvents were dried by standard procedures. Diols $\mathbf{1 a},{ }^{10} \mathbf{1} \mathbf{b}^{11}$ and $\mathbf{1} \mathbf{c}^{12}$ were obtained according to the literature procedures. NMR spectra were recorded on a Bruker DPX 250 instrument at $250.13 \mathrm{MHz}$ for ${ }^{1} \mathrm{H}$ and $62.9 \mathrm{MHz}$ for ${ }^{13} \mathrm{C}$ and $101.3 \mathrm{MHz}$ for ${ }^{31} \mathrm{P} \mathrm{NMR}$, respectively, using tetramethylsilane as internal and $85 \% \mathrm{H}_{3} \mathrm{PO}_{4}$ as external standard. The multiplicities of carbons were determined by DEPT experiments. IR spectra were measured on Specord M80 (Zeiss) instrument. Elemental analyses were performed on Perkin-Elmer PE 2400 analyzer. Melting points were determined in open capillaries and were uncorrected.

\section{X-ray crystal structure analysis}

A colourless single crystal of $\mathbf{5}(0.1 \times 0.2 \times 0.6 \mathrm{~mm})$ was obtained by a slow evaporation from the chloroform-acetone mixture. X-ray data were collected on the Bruker Smart APEX diffractometer at room temperature with a graphite monochromatized MoKa radiation. Crystal structure was solved with direct methods and further refined using full matrix least squares technique. Crystal data and structure analysis parameters are summarized in Table 1. The following computer programs were applied during the analysis: data collection SMART, ${ }^{17}$ data reduction SAINT-PLUS ${ }^{18}$ absorption correction $S A D A B S,{ }^{19}$ structure solution, refinement, and molecular graphics SHELXTL. ${ }^{20}$

Crystallographic data (excluding structure factors) for the structure reported in this article have been deposited with the Cambridge Crystallographic Data Center as supplementary publication no. CCDC 764399. Copies of the data can be obtained free of charge on application 
to CCDC, 12 Union Road, Cambridge CB2 1EZ, UK. Any request should be accompanied by a full literature citation.

Table 1. Crystal data and structural refinement details for $\mathbf{5}$

\begin{tabular}{|c|c|}
\hline Parameters & 5 \\
\hline Empirical formula & $\mathrm{C}_{16} \mathrm{H}_{23} \mathrm{O}_{6} \mathrm{P}$ \\
\hline Formula weight & 356.32 \\
\hline Temperature & $293(2) \mathrm{K}$ \\
\hline Wavelength & $0.71073 \AA$ \\
\hline Crystal system, space group & monoclinic, $P 2_{1}$ \\
\hline Unit cell dimensions & $\begin{array}{c}a=10.7927(9), b=7.0051(5), \\
c=12.4943(10) \AA, \beta=113.107(3)^{\circ}\end{array}$ \\
\hline$Z$ & 2 \\
\hline Unit cell volume & $V=868.83(12) \AA^{3}$ \\
\hline Density (calculated) & $1.362 \mathrm{~g} \mathrm{~cm}^{-3}$ \\
\hline Absorption coefficient & $0.19 \mathrm{~mm}^{-1}$ \\
\hline $\mathrm{F}(000)$ & 378.0 \\
\hline Crystal size & $0.1 \times 0.2 \times 0.6 \mathrm{~mm}$ \\
\hline Max. theta for the data collection & $25.0^{\circ}$ \\
\hline Index ranges & $-12 \leq h \leq 12,-8 \leq k \leq 8,-14 \leq l \leq 14$ \\
\hline Number of collected reflections & 20942 \\
\hline Number of independent reflections & $3069\left(R_{\mathrm{int}}=0.023\right)$ \\
\hline Absorption correction & multi-scan (Sadabs) \\
\hline Refinement method & Full-matrix least-squares on $F^{2}$ \\
\hline Data/restraints/parameters & $3069 / 0 / 258$ \\
\hline Goodness of fit on $\mathrm{F}^{2}$ & 1.071 \\
\hline Final $R$ indices $[I>2 \sigma(I)]$ & $R_{1}=0.0542, w R^{2}=0.1490$ \\
\hline $\mathrm{R}$ indices (all data) & $R_{1}=0.0547, w R^{2}=0.1504$ \\
\hline $\begin{array}{l}\text { Max. and min. on a difference Fourier } \\
\text { map }\end{array}$ & 0.474 and $-0.302 \mathrm{e}^{-3}$ \\
\hline
\end{tabular}

\section{General procedure for preparation 2,2-Dioxo-1,3,2-dioxathiolanes (2a-d)}

(Dioxathiolanes 2a-d were obtained by general procedure) ${ }^{1}$

2,2-Dioxo-1,3,2-dioxathiolane-4-carboxylic acid ethyl ester (2a). Yield: (90\%); yellowish oil. ${ }^{1} \mathrm{H}$ NMR $\left(\mathrm{CDCl}_{3}\right): \delta 1.36\left(\mathrm{t},{ }^{3} \mathrm{~J}=7.2 \mathrm{~Hz}, 3 \mathrm{H}, \mathrm{CH}_{3} \mathrm{CH}_{2} \mathrm{O}\right) ; 4.37\left(\mathrm{q},{ }^{3} \mathrm{~J}=7.2 \mathrm{~Hz}, 2 \mathrm{H}, \mathrm{CH}_{2} \mathrm{O}\right) ; 4.84$ $\left(\mathrm{dd},{ }^{2} J=9.0 \mathrm{~Hz},{ }^{3} \mathrm{~J}=5.7 \mathrm{~Hz}, 1 \mathrm{H}, \mathrm{CHCHH}\right) ; 4.92\left(\mathrm{dd},{ }^{2} \mathrm{~J}=9.0 \mathrm{~Hz},{ }^{3} \mathrm{~J}=7.2 \mathrm{~Hz}, 1 \mathrm{H}, \mathrm{CHCH}\right)$; $5.27\left(\mathrm{dd},{ }^{3} J=7.2 \mathrm{~Hz},{ }^{3} J=5.7 \mathrm{~Hz}, 1 \mathrm{H}, \mathrm{CHCH}_{2}\right) .{ }^{13} \mathrm{C} \mathrm{NMR}\left(\mathrm{CDCl}_{3}\right): \delta 13.62\left(\mathrm{~s}, \mathrm{CH}_{3} \mathrm{CH}_{2} \mathrm{O}\right)$; $63.21\left(\mathrm{~s}, \mathrm{CH}_{2} \mathrm{O}\right) ; 69.77\left(\mathrm{~s}, \mathrm{CHCH}_{2}\right) ; 75.80\left(\mathrm{~s}, \mathrm{CHCH}_{2}\right) ; 164.95$ (s, C=O). IR (film) v(C=O) 1752.

4-Diethoxymethyl-2,2-dioxo-1,3,2-dioxathiolane (2b). Yield: (70\%); yellow oil. ${ }^{1} \mathrm{H}$ NMR $\left(\mathrm{CDCl}_{3}\right): \delta 1.24\left(\mathrm{t},{ }^{3} \mathrm{~J}=7.0 \mathrm{~Hz}, 3 \mathrm{H}, \mathrm{CH}_{3} \mathrm{CH}_{2} \mathrm{O}\right) ; 1.26\left(\mathrm{t},{ }^{3} \mathrm{~J}=7.0 \mathrm{~Hz}, 3 \mathrm{H}, \mathrm{CH}_{3} \mathrm{CH}_{2} \mathrm{O}\right) ; 3.58 \div 3.82$ 
$\left(\mathrm{m}, 5 \mathrm{H}, \mathrm{CH}_{2} \mathrm{O}, \mathrm{CH}_{2} \mathrm{CHCH}, \mathrm{CH}_{2} \mathrm{CH}\right) ; 3.98 \div 4.05\left(\mathrm{~m}, 2 \mathrm{H}, \mathrm{CH}_{2} \mathrm{O}\right) ; 4.67\left(\mathrm{~d},{ }^{3} \mathrm{~J}=5.0 \mathrm{~Hz}, 1 \mathrm{H}\right.$, $\left.\mathrm{CH}_{2} \mathrm{CHCH}\right) .{ }^{13} \mathrm{C}$ NMR $\left(\mathrm{MHz}, \mathrm{CDCl}_{3}\right): \delta 14.82\left(\mathrm{~s}, \mathrm{CH}_{3} \mathrm{CH}_{2} \mathrm{O}\right) ; 14.86\left(\mathrm{~s}, \mathrm{CH}_{3} \mathrm{CH}_{2} \mathrm{O}\right) ; 64.22(\mathrm{~s}$, $\left.\mathrm{CH}_{2} \mathrm{O}\right) ; 65.18$ (s, $\left.\mathrm{CH}_{2} \mathrm{O}\right) ; 68.17$ (s, $\left.\mathrm{CH}_{2} \mathrm{CHCH}\right) ; 79.57$ (s, $\left.\mathrm{CH}_{2} \mathrm{CHCH}\right) ; 99.92$ (s, $\left.\mathrm{CH}_{2} \mathrm{CHCH}\right)$.

4-Benzyloxymethyl-2,2-dioxo-1,3,2-dioxathiolane (2c). ${ }^{2}$ Yield: (85\%); yellowish oil. ${ }^{1} \mathrm{H}$ NMR $\left(\mathrm{CDCl}_{3}\right): \delta 3.78\left(\mathrm{~d},{ }^{3} \mathrm{~J}=5.2 \mathrm{~Hz}, 2 \mathrm{H}, \mathrm{CHCH}_{2} \mathrm{OBn}\right) ; 4.50 \div 4.76\left(\mathrm{~m}, 4 \mathrm{H}, \mathrm{CH}_{2} \mathrm{Ph}, \mathrm{CHCH}_{2} \mathrm{O}\right) ; 4.99$ $\div 5.10(\mathrm{~m}, 1 \mathrm{H}, \mathrm{CHO}) ; 7.33 \div 7.41(\mathrm{~m}, 5 \mathrm{H}, \mathrm{CH}$ Ar).

4-Butyl-2,2-dioxo-1,3,2-dioxathiolane (2d). ${ }^{13}$. Yield: ( $\left.95 \%\right)$; yellowish oil. ${ }^{1} \mathrm{H} \mathrm{NMR}\left(\mathrm{CDCl}_{3}\right)$ : $\delta 0.94\left(\mathrm{t},{ }^{3} \mathrm{~J}=6.5 \mathrm{~Hz}, 3 \mathrm{H}, \mathrm{CH}_{3} \mathrm{CH}_{2}\right) ; 1.35 \div 1.54\left(\mathrm{~m}, 4 \mathrm{H},\left(\mathrm{CH}_{2}\right)_{2}\right) ; 1.70 \div 1.81(\mathrm{~m}, 1 \mathrm{H}$, $\left.\mathrm{CH}_{2} \mathrm{CHHCHO}\right) ; 1.89 \div 2.02\left(\mathrm{~m}, 1 \mathrm{H}, \mathrm{CH}_{2} \mathrm{CH} H \mathrm{CHO}\right) ; 4.34\left(\mathrm{dd},{ }^{2} \mathrm{~J}=8.5 \mathrm{~Hz},{ }^{3} \mathrm{~J}=8.2 \mathrm{~Hz}, 1 \mathrm{H}\right.$, $\mathrm{CHCH} H \mathrm{O}) ; 4.71\left(\mathrm{dd},{ }^{2} J=8.5 \mathrm{~Hz},{ }^{3} J=6.0 \mathrm{~Hz}, 1 \mathrm{H}, \mathrm{CHCHHO}\right) ; 4.92 \div 5.03(\mathrm{~m}, 1 \mathrm{H}, \mathrm{CHO})$.

\section{General procedure for preparation 1-(diethoxyphosphoryl)cyclopropanecarboxylates 4a-d}

To a stirred suspension of $\mathrm{NaH}(0.15 \mathrm{~g}, 6.0 \mathrm{mmol})$ in THF $(25 \mathrm{~mL})$ triethylphosphonoacetate 3 $(0.60 \mathrm{~mL}, 3.0 \mathrm{mmol})$ was added at room temperature. After stirring for $0.5 \mathrm{~h}$ a solution of corresponding sulfate $2(3.0 \mathrm{mmol})$ in THF $(10 \mathrm{~mL})$ was added and the resulting mixture was stirred at room temperature for $0.5 \mathrm{~h}$ and then at reflux for $8 \mathrm{~h}$. After that time, the reaction mixture was poured into water $(5 \mathrm{~mL})$, extracted with DCM $(3 \times 10 \mathrm{~mL})$ and organic layer was dried over $\mathrm{MgSO}_{4}$. Removal of the solvent gave the crude products 4 , which were purified by column chromatography ( silica gel, eluent: chloroform/acetone 80:20).

trans-Diethyl 1-(diethoxyphosphoryl)cyclopropane-1,2-dicarboxylate (4a). Yield: $0.62 \mathrm{~g}$ $(64 \%)$; colorless oil. $\mathrm{R}_{\mathrm{f}}=0.30 .{ }^{31} \mathrm{P} \mathrm{NMR}\left(\mathrm{C}_{6} \mathrm{D}_{6}\right): \delta 17.58 .{ }^{1} \mathrm{H} \mathrm{NMR}\left(\mathrm{C}_{6} \mathrm{D}_{6}\right): \delta 1.26\left(\mathrm{t},{ }^{3} J_{\mathrm{HH}}=7.0\right.$ $\left.\mathrm{Hz}, 3 \mathrm{H}, \mathrm{CH}_{3} \mathrm{CH}_{2} \mathrm{O}\right) ; 1.27\left(\mathrm{t},{ }^{3} J_{\mathrm{HH}}=7.0 \mathrm{~Hz}, 3 \mathrm{H}, \mathrm{CH}_{3} \mathrm{CH}_{2} \mathrm{O}\right) ; 1.35\left(\mathrm{td},{ }^{3} J_{\mathrm{HH}}=7.0 \mathrm{~Hz},{ }^{4} J_{\mathrm{PH}}=0.7\right.$ $\left.\mathrm{Hz}, 3 \mathrm{H}, \mathrm{CH}_{3} \mathrm{CH}_{2} \mathrm{OP}\right) ; 1.37\left(\mathrm{td},{ }^{3} J_{\mathrm{HH}}=7.0 \mathrm{~Hz},{ }^{4} J_{\mathrm{PH}}=0.7 \mathrm{~Hz}, 3 \mathrm{H}, \mathrm{CH}_{3} \mathrm{CH}_{2} \mathrm{OP}\right) ; 1.70\left(\mathrm{ddd},{ }^{3} J_{\mathrm{PH}}=\right.$ $\left.16.2 \mathrm{~Hz},{ }^{3} J_{\mathrm{HH}}=8.5 \mathrm{~Hz},{ }^{2} J_{\mathrm{HH}}=4.5 \mathrm{~Hz}, 1 \mathrm{H}, \mathrm{PCCHH}\right) ; 1.92\left(\mathrm{ddd},{ }^{3} J_{\mathrm{PH}}=13.0 \mathrm{~Hz},{ }^{3} J_{\mathrm{HH}}=6.2 \mathrm{~Hz}\right.$, $\left.{ }^{2} J_{\mathrm{HH}}=4.0 \mathrm{~Hz}, 1 \mathrm{H}, \mathrm{PCCH}\right) ; 2.45\left(\mathrm{ddd},{ }^{3} J_{\mathrm{PH}}=16.0 \mathrm{~Hz},{ }^{3} J_{\mathrm{HH}}=8.5 \mathrm{~Hz},{ }^{3} J_{\mathrm{HH}}=6.2 \mathrm{~Hz}, 1 \mathrm{H}\right.$, $\mathrm{PCCH}) ; 4.10 \div 4.25\left(\mathrm{~m}, 8 \mathrm{H}, 2 \times \mathrm{CH}_{2} \mathrm{OP}, 2 \times \mathrm{CH}_{2} \mathrm{O}\right) .{ }^{13} \mathrm{C} \mathrm{NMR}\left(\mathrm{CDCl}_{3}\right): \delta 13.49\left(\mathrm{~s}, \mathrm{CH}_{3} \mathrm{CH}_{2} \mathrm{O}\right)$; $13.64\left(\mathrm{~s}, \mathrm{CH}_{3} \mathrm{CH}_{2} \mathrm{O}\right) ; 15.78\left(\mathrm{~d},{ }^{3} \mathrm{~J}_{\mathrm{PC}}=6.0 \mathrm{~Hz}, \mathrm{CH}_{3} \mathrm{CH}_{2} \mathrm{OP}\right) ; 15.83\left(\mathrm{~s}, \mathrm{PCCH}_{2}\right) ; 24.17$ (s, $\left.\mathrm{PCCH}\right)$; $28.70\left(\mathrm{~d},{ }^{1} \mathrm{~J}_{\mathrm{PC}}=177.95 \mathrm{~Hz}, \mathrm{PC}\right) ; 60.95\left(\mathrm{~s}, \mathrm{CH}_{2} \mathrm{O}\right) ; 61.42\left(\mathrm{~s}, \mathrm{CH}_{2} \mathrm{O}\right) ; 62.88\left(\mathrm{~s}, \mathrm{CH}_{2} \mathrm{OP}\right) ; 62.90(\mathrm{~d}$, $\left.{ }^{2} J_{\mathrm{PC}}=5.60 \mathrm{~Hz}, C \mathrm{H}_{2} \mathrm{OP}\right) ; 165.70\left(\mathrm{~d},{ }^{2} J_{\mathrm{PC}}=4.9 \mathrm{~Hz}, C=\mathrm{O}\right) ; 168.81\left(\mathrm{~d},{ }^{3} J_{\mathrm{PC}}=4.0 \mathrm{~Hz}, C=\mathrm{O}\right)$. IR (film) $v_{(\mathrm{C}=\mathrm{O})} 1740, v_{(\mathrm{P}=\mathrm{O})} 1220, v_{(\mathrm{P}-\mathrm{O})}$ 1024. Anal. Calcd. for $\mathrm{C}_{13} \mathrm{H}_{23} \mathrm{O}_{7} \mathrm{P}: \mathrm{C} 48.45, \mathrm{H}$ 7.19. Found: C 48.36, H 7.21.

trans-Ethyl 2-(diethoxymethyl)-1-(diethoxyphosphoryl)cyclopropanecarboxylate

(4b). Yield: $0.64 \mathrm{~g}(60 \%)$; colorless oil. $\mathrm{R}_{\mathrm{f}}=0.32 .{ }^{31} \mathrm{P} \mathrm{NMR}\left(\mathrm{C}_{6} \mathrm{D}_{6}\right): \delta 23.08 .{ }^{1} \mathrm{H} \mathrm{NMR}\left(\mathrm{C}_{6} \mathrm{D}_{6}\right): \delta 1.22 \div$ $1.33\left(\mathrm{~m}, 6 \mathrm{H}, 2 \times \mathrm{CH}_{3} \mathrm{CH}_{2} \mathrm{O}\right) ; 1.34\left(\mathrm{t},{ }^{3} J_{\mathrm{HH}}=7.0 \mathrm{~Hz}, 6 \mathrm{H}, 2 \mathrm{xCH}_{3} \mathrm{CH}_{2} \mathrm{OP}\right) ; 1.43\left(\mathrm{t},{ }^{3} J_{\mathrm{HH}}=7.0 \mathrm{~Hz}\right.$, $\left.3 \mathrm{H}, \mathrm{CH}_{3} \mathrm{CH}_{2} \mathrm{O}\right) ; 1.80 \div 1.90\left(\mathrm{~m}, 3 \mathrm{H}, \mathrm{PCCH}_{2}, \mathrm{PCCH}\right) ; 3.67 \div 3.84\left(\mathrm{~m}, 4 \mathrm{H}, 2 \times \mathrm{CH}_{2} \mathrm{O}\right) ; 4.00 \div 4.25$ $\left(\mathrm{m}, 4 \mathrm{H}, 2 \mathrm{xCH} \mathrm{H}_{2} \mathrm{OP}\right) ; 4.34\left(\mathrm{q},{ }^{3} J_{\mathrm{HH}}=7.0 \mathrm{~Hz}, 2 \mathrm{H}, \mathrm{CH}_{2} \mathrm{O}\right) ; 4.68\left(\mathrm{~d},{ }^{3} J_{\mathrm{HH}}=7.0 \mathrm{~Hz}, \mathrm{OCHO}\right) .{ }^{13} \mathrm{C}$ NMR $\left(\mathrm{CDCl}_{3}\right): \delta 13.72\left(\mathrm{~s}, \mathrm{CH}_{3} \mathrm{CH}_{2} \mathrm{O}\right) ; 14.77\left(\mathrm{~s}, \mathrm{CH}_{3} \mathrm{CH}_{2} \mathrm{O}\right) ; 14.80\left(\mathrm{~s}, \mathrm{CH}_{3} \mathrm{CH}_{2} \mathrm{O}\right) ; 15.91$ (s, $\mathrm{CH}_{3} \mathrm{CH}_{2} \mathrm{OP}$ ); 15.99 (s, $\left.\mathrm{CH}_{3} \mathrm{CH}_{2} \mathrm{OP}\right) ; 20.26\left(\mathrm{~d},{ }^{2} \mathrm{~J}_{\mathrm{PC}}=4.9 \mathrm{~Hz}, \mathrm{PCCH}\right) ; 21.71\left(\mathrm{~d},{ }^{1} \mathrm{~J}_{\mathrm{PC}}=178.4 \mathrm{~Hz}\right.$, $\mathrm{PC}) ; 27.38\left(\mathrm{~s}, \mathrm{PCCH}_{2}\right) ; 61.35\left(\mathrm{~s}, \mathrm{CH}_{2} \mathrm{O}\right) ; 62.22\left(2 \mathrm{~d},{ }^{2} \mathrm{~J}_{\mathrm{PC}}=5.8 \mathrm{~Hz}, \mathrm{CH}_{2} \mathrm{OP}\right) ; 63.56\left(\mathrm{~s}, \mathrm{CH}_{2} \mathrm{O}\right)$; 
$64.89\left(\mathrm{~s}, \mathrm{CH}_{2} \mathrm{O}\right) ; 79.34(\mathrm{~s}, \mathrm{OCHO}) ; 168.73\left(\mathrm{~d},{ }^{2} J_{\mathrm{PC}}=4.5 \mathrm{~Hz}, \mathrm{C}=\mathrm{O}\right)$. IR (film) $v_{(\mathrm{C}=\mathrm{O})} 1748, v_{(\mathrm{P}=\mathrm{O})}$ 1264, $v_{(\mathrm{P}-\mathrm{O})}$ 1024. Anal. Calcd. for $\mathrm{C}_{15} \mathrm{H}_{29} \mathrm{O}_{7} \mathrm{P}: \mathrm{C} 51.13, \mathrm{H}$ 8.30. Found: C 51.26, H 8.34.

trans-Ethyl 2-(benzyloxymethyl)-1-(diethoxyphosphoryl)cyclopropanecarboxylate (4c). Yield: $0.70 \mathrm{~g}(63 \%)$; colorless oil. $\mathrm{R}_{\mathrm{f}}=0.38 .{ }^{31} \mathrm{P}$ NMR $\left(\mathrm{C}_{6} \mathrm{D}_{6}\right): \delta 23.14 .{ }^{1} \mathrm{H}$ NMR $\left(\mathrm{C}_{6} \mathrm{D}_{6}\right): \delta 1.23$ $\left(\mathrm{t},{ }^{3} J_{\mathrm{HH}}=7.2 \mathrm{~Hz}, 3 \mathrm{H}, \mathrm{CH}_{3} \mathrm{CH}_{2} \mathrm{O}\right) ; 1.31\left(\mathrm{t},{ }^{3} J_{\mathrm{HH}}=7.0 \mathrm{~Hz}, 6 \mathrm{H}, 2 \mathrm{xCH}_{3} \mathrm{CH}_{2} \mathrm{OP}\right) ; 1.54 \div 1.68(\mathrm{~m}$, $\left.2 \mathrm{H}, \mathrm{PCCH}_{2}\right) ; 2.12 \div 2.27(\mathrm{~m}, 1 \mathrm{H}, \mathrm{PCCH}) ; 3.48\left(\mathrm{dd},{ }^{2} J_{\mathrm{HH}}=10.5 \mathrm{~Hz},{ }^{3} \mathrm{~J}_{\mathrm{HH}}=8.7 \mathrm{~Hz}, 1 \mathrm{H}\right.$, $\left.\mathrm{CHHOCH}_{2} \mathrm{Ph}\right) ; 3.78\left(\mathrm{dd},{ }^{2} J_{\mathrm{HH}}=10.5 \mathrm{~Hz},{ }^{3} J_{\mathrm{HH}}=5.5 \mathrm{~Hz}, 1 \mathrm{H}, \mathrm{CH} \mathrm{OCH}_{2} \mathrm{Ph}\right) ; 4.11 \div 4.23(\mathrm{~m}, 6 \mathrm{H}$,

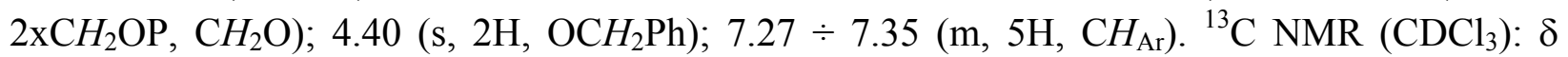
$13.87\left(\mathrm{~s}, \mathrm{CH}_{3} \mathrm{CH}_{2} \mathrm{O}\right) ; 16.10\left(\mathrm{~d},{ }^{3} \mathrm{~J}_{\mathrm{PC}}=6.7 \mathrm{~Hz}, \mathrm{CH}_{3} \mathrm{CH}_{2} \mathrm{OP}\right) ; 16.20\left(\mathrm{~d},{ }^{3} J_{\mathrm{PC}}=6.1 \mathrm{~Hz}, \mathrm{CH}_{3} \mathrm{CH}_{2} \mathrm{OP}\right)$; $16.58\left(\mathrm{~s}, \mathrm{PCCH}_{2}\right) ; 23.77\left(\mathrm{~d},{ }^{1} J_{\mathrm{PC}}=188.5 \mathrm{~Hz}, \mathrm{PC}\right) ; 26.31\left(\mathrm{~d},{ }^{2} J_{\mathrm{PC}}=2.8 \mathrm{~Hz}, \mathrm{PCCH}\right) ; 61.43(\mathrm{~s}$, $\left.\mathrm{CH}_{2} \mathrm{O}\right) ; 62.47\left(\mathrm{~d},{ }^{2} \mathrm{~J}_{\mathrm{PC}}=6.2 \mathrm{~Hz}, \mathrm{CH}_{2} \mathrm{OP}\right) ; 62.57\left(\mathrm{~d},{ }^{2} \mathrm{~J}_{\mathrm{PC}}=6.5 \mathrm{~Hz}, \mathrm{CH}_{2} \mathrm{OP}\right) ; 67.40\left(\mathrm{~s}, \mathrm{CHCH}_{2} \mathrm{O}\right)$; $72.42\left(\mathrm{~s}, \mathrm{OCH}_{2} \mathrm{Ph}\right) ; 127.20\left(\mathrm{~s}, 2 \times \mathrm{CH}_{\mathrm{Ar}}\right) ; 127.37\left(\mathrm{~s}, \mathrm{CH}_{\mathrm{Ar}}\right) ; 128.10\left(\mathrm{~s}, 2 \times \mathrm{CH}_{\mathrm{Ar}}\right) ; 136.40\left(\mathrm{~s}, C_{\mathrm{Ar}}\right)$; $166.27(\mathrm{~s}, C=\mathrm{O})$. IR (film) $\mathrm{v}_{(\mathrm{C}=\mathrm{O})} 1724, \mathrm{v}_{(\mathrm{P}=\mathrm{O})} 1270, \mathrm{v}_{(\mathrm{P}-\mathrm{O})}$ 1028. Anal. Calcd. for $\mathrm{C}_{18} \mathrm{H}_{27} \mathrm{O}_{6} \mathrm{P}: \mathrm{C}$ 58.37, H 7.35. Found: C, 58.49; H, 7.31.

trans-Ethyl 2-(butyl)-1-(diethoxyphosphoryl)cyclopropanecarboxylate (4d). Yield: $0.58 \mathrm{~g}$ $(63 \%)$; colorless oil. $\mathrm{R}_{\mathrm{f}}=0.48 .{ }^{31} \mathrm{P} \mathrm{NMR}\left(\mathrm{C}_{6} \mathrm{D}_{6}\right): \delta 24.30 .{ }^{1} \mathrm{H} \mathrm{NMR}\left(\mathrm{C}_{6} \mathrm{D}_{6}\right): \delta 0.89\left(\mathrm{t},{ }^{3} J_{\mathrm{HH}}=7.0\right.$ $\left.\mathrm{Hz}, 3 \mathrm{H}, \mathrm{CH}_{3} \mathrm{CH}_{2}\right) ; 1.29\left(\mathrm{t},{ }^{3} J_{\mathrm{HH}}=7.2 \mathrm{~Hz}, 3 \mathrm{H}, \mathrm{CH}_{3} \mathrm{CH}_{2} \mathrm{O}\right) ; 1.34\left(\mathrm{t},{ }^{3} J_{\mathrm{HH}}=7.0 \mathrm{~Hz}, 3 \mathrm{H}\right.$, $\left.\mathrm{CH}_{3} \mathrm{CH}_{2} \mathrm{OP}\right) ; 1.35\left(\mathrm{t},{ }^{3} \mathrm{~J}_{\mathrm{HH}}=7.0 \mathrm{~Hz}, 3 \mathrm{H}, \mathrm{CH}_{3} \mathrm{CH}_{2} \mathrm{OP}\right) ; 1.23 \div 1.60\left(\mathrm{~m}, 9 \mathrm{H},\left(\mathrm{CH}_{2}\right)_{3}, \mathrm{PCCH}_{2}\right.$, $\mathrm{PCCH}) ; 4.14\left(\mathrm{dq},{ }^{3} J_{\mathrm{PH}}=7.0 \mathrm{~Hz},{ }^{3} J_{\mathrm{HH}}=7.0 \mathrm{~Hz}, 2 \mathrm{H}, \mathrm{CH}_{2} \mathrm{OP}\right) ; 4.15\left(\mathrm{dq},{ }^{3} J_{\mathrm{PH}}=7.0 \mathrm{~Hz},{ }^{3} J_{\mathrm{HH}}=7.0\right.$ $\left.\mathrm{Hz}, 2 \mathrm{H}, \mathrm{CH}_{2} \mathrm{OP}\right) ; 4.16\left(\mathrm{q},{ }^{3} J_{\mathrm{HH}}=7.2 \mathrm{~Hz}, 1 \mathrm{H}, \mathrm{CHHO}\right) ; 4.21\left(\mathrm{q},{ }^{3} J_{\mathrm{HH}}=7.2 \mathrm{~Hz}, 1 \mathrm{H}, \mathrm{CH} H \mathrm{O}\right) .{ }^{13} \mathrm{C}$ NMR $\left(\mathrm{CDCl}_{3}\right): \delta 13.62\left(\mathrm{~s}, \mathrm{CH}_{3} \mathrm{CH}_{2}\right) ; 13.90\left(\mathrm{~s}, \mathrm{CH}_{3} \mathrm{CH}_{2} \mathrm{O}\right) ; 16.09\left(\mathrm{~d},{ }^{3} \mathrm{~J}_{\mathrm{PC}}=6.1 \mathrm{~Hz}\right.$, $\left.\left.\mathrm{CH}_{3} \mathrm{CH}_{2} \mathrm{O}\right) \mathrm{P}\right) ; 18.17\left(\mathrm{~d},{ }^{2} \mathrm{~J}_{\mathrm{PC}}=2.8 \mathrm{~Hz}, \mathrm{PCCH}_{2}\right) ; 21.93\left(\mathrm{~s}, \mathrm{CH}_{3} \mathrm{CH}_{2}\right) ; 24.96\left(\mathrm{~d},{ }^{1} J_{\mathrm{PC}}=189.5 \mathrm{~Hz}\right.$, PC); $26.96\left(\mathrm{~s}, \mathrm{CH}_{3} \mathrm{CH}_{2} \mathrm{CH}_{2}\right) ; 27.03(\mathrm{~s}, \mathrm{PCCH}) ; 30.87\left(\mathrm{~s}, \mathrm{CH}_{2} \mathrm{CH}_{2} \mathrm{CH}\right) ; 61.05\left(\mathrm{~s}, \mathrm{CH}_{2} \mathrm{O}\right) ; 62.08$ $\left(\mathrm{d},{ }^{2} J_{\mathrm{PC}}=6.2 \mathrm{~Hz}, \mathrm{CH}_{2} \mathrm{OP}\right) ; 62.18\left(\mathrm{~d},{ }^{2} J_{\mathrm{PC}}=6.3 \mathrm{~Hz}, C \mathrm{H}_{2} \mathrm{OP}\right) ; 168.04\left(\mathrm{~d},{ }^{2} J_{\mathrm{PC}}=7.2 \mathrm{~Hz}, C=\mathrm{O}\right)$. IR (film) $v_{(\mathrm{C}=\mathrm{O})} 1724, \mathrm{v}_{(\mathrm{P}=\mathrm{O})} 1252, \mathrm{v}_{(\mathrm{P}-\mathrm{O})}$ 1028. Anal. Calcd. for $\mathrm{C}_{14} \mathrm{H}_{27} \mathrm{O} 5 \mathrm{P}: \mathrm{C} 54.89, \mathrm{H}$ 8.88. Found C 55.00, H 8.84.

Procedure for preparation trans-2-Benzyloxymethyl-1-(diethoxyphosphoryl)cyclopropanecarboxylic acid (5)

To a solution of cyclopropanecarboxylate $4 \mathrm{c}(0.37 \mathrm{~g}, 1.00 \mathrm{mmol})$ in ethyl alcohol $(5 \mathrm{~mL}) \mathrm{a}$ solution of $\mathrm{NaOH}(0.08 \mathrm{~g} ; 2.00 \mathrm{mmol})$ in water $(0.5 \mathrm{~mL})$ was added and the reaction mixture was stirred at room temperature for 2 days. Then the solvent was evaporated and residue was dissolved in water $(10 \mathrm{~mL})$ and extracted with diethyl ether $(3 \times 10 \mathrm{~mL})$. Then the water layer was acidified to $\mathrm{pH} 1$ with $3 \mathrm{~N} \mathrm{HCl}$ and extracted with dichloromethane $(3 \times 10 \mathrm{~mL})$. The combined organic layers were dried $\left(\mathrm{MgSO}_{4}\right)$ and evaporated under reduced pressure. The residue crystallized on standby to give acid $\mathbf{5}$ as white solid, which was collected by filtration from diethyl ether.

trans-2-(Benzyloxymethyl)-1-(diethoxyphosphoryl)cyclopropanecarboxylic acid (5). Yield: 0.33 g (97\%); white crystal; m.p. $=102-104{ }^{\circ} \mathrm{C} .{ }^{31} \mathrm{P} \mathrm{NMR}\left(\mathrm{CDCl}_{3}\right): \delta 27.42 .{ }^{1} \mathrm{H}$ NMR $\left(\mathrm{CDCl}_{3}\right): \delta$ $1.30\left(\mathrm{td},{ }^{3} J_{\mathrm{HH}}=7.0 \mathrm{~Hz},{ }^{4} J_{\mathrm{PH}}=0.7 \mathrm{~Hz}, 6 \mathrm{H}, 2 \mathrm{xCH} \mathrm{CH}_{2} \mathrm{OP}\right) ; 1.61\left(\mathrm{dd},{ }^{3} J_{\mathrm{PH}}=12.2 \mathrm{~Hz},{ }^{3} J_{\mathrm{HH}}=8.5\right.$ 
$\mathrm{Hz}, 2 \mathrm{H}, \mathrm{PCCH} \mathrm{H}_{2}$ ); $2.23\left(\mathrm{ddddd},{ }^{3} J_{\mathrm{PH}}=16.5 \mathrm{~Hz},{ }^{3} J_{\mathrm{HH}}=10.0 \mathrm{~Hz},{ }^{3} J_{\mathrm{HH}}=8.5 \mathrm{~Hz},{ }^{3} J_{\mathrm{HH}}=8.5 \mathrm{~Hz}\right.$, $\left.{ }^{3} J_{\mathrm{HH}}=5.7 \mathrm{~Hz} 1 \mathrm{H}, \mathrm{PCCH}\right) ; 3.55\left(\mathrm{dd},{ }^{2} J_{\mathrm{HH}}=10.7 \mathrm{~Hz},{ }^{3} J_{\mathrm{HH}}=8.5 \mathrm{~Hz}, 1 \mathrm{H}, \mathrm{CHHOCH}{ }_{2} \mathrm{Ph}\right) ; 3.78(\mathrm{dd}$,

$\left.{ }^{2} J_{\mathrm{HH}}=10.7 \mathrm{~Hz},{ }^{3} J_{\mathrm{HH}}=5.7 \mathrm{~Hz}, 1 \mathrm{H}, \mathrm{CH} H \mathrm{OCH}_{2} \mathrm{Ph}\right) ; 4.16\left(\mathrm{dq},{ }^{3} J_{\mathrm{PH}}={ }^{3} J_{\mathrm{HH}}=7.0 \mathrm{~Hz}, 1 \mathrm{H}, \mathrm{CHHOP}\right)$; $4.17\left(\mathrm{dq},{ }^{3} J_{\mathrm{PH}}={ }^{3} J_{\mathrm{HH}}=7.0 \mathrm{~Hz}, 1 \mathrm{H}, \mathrm{CH} H \mathrm{OP}\right) ; 4.18\left(\mathrm{dq},{ }^{3} J_{\mathrm{PH}}={ }^{3} J_{\mathrm{HH}}=7.00 \mathrm{~Hz}, 2 \mathrm{H}, \mathrm{CH}_{2} \mathrm{OP}\right) ; 4.47$ $\left(\mathrm{s}, 2 \mathrm{H}, \mathrm{OCH}_{2} \mathrm{Ph}\right) ; 7.25 \div 7.32\left(\mathrm{~m}, 5 \mathrm{H}, \mathrm{CH}_{\mathrm{Ar}}\right) \cdot{ }^{13} \mathrm{C} \mathrm{NMR}\left(\mathrm{CDCl}_{3}\right): \delta 16.04\left(\mathrm{~d},{ }^{3} J_{\mathrm{PC}}=6.1 \mathrm{~Hz}\right.$, $\left.\mathrm{CH}_{3} \mathrm{CH}_{2} \mathrm{OP}\right) ; 16.13\left(\mathrm{~d},{ }^{3} \mathrm{~J}_{\mathrm{PC}}=5.0 \mathrm{~Hz}, \mathrm{CH}_{3} \mathrm{CH}_{2} \mathrm{OP}\right) ; 17.24\left(\mathrm{~s}, \mathrm{PCCH}_{2}\right) ; 23.38\left(\mathrm{~d},{ }^{1} J_{\mathrm{PC}}=190.9 \mathrm{~Hz}\right.$, $\mathrm{PC}) ; 26.95(\mathrm{~s}, \mathrm{PCCH}) ; 63.01\left(\mathrm{~d},{ }^{2} J_{\mathrm{PC}}=6.5 \mathrm{~Hz}, \mathrm{CH}_{2} \mathrm{OP}\right) ; 63.11\left(\mathrm{~d},{ }^{2} J_{\mathrm{PC}}=6.5 \mathrm{~Hz}, \mathrm{CH}_{2} \mathrm{OP}\right) ; 67.22$ (s, $\left.\mathrm{CH}_{2} \mathrm{OCH}_{2} \mathrm{Ph}\right) ; 72.43\left(\mathrm{~s}, \mathrm{OCH}_{2} \mathrm{Ph}\right) ; 127.36\left(\mathrm{~s}, 2 \times \mathrm{CH}_{\mathrm{Ar}}\right) ; 128.13\left(\mathrm{~s}, \mathrm{CH}_{\mathrm{Ar}}\right) ; 128.14\left(\mathrm{~s}, 2 \times C \mathrm{H}_{\mathrm{Ar}}\right)$; $137.94\left(\mathrm{~s}, C_{\mathrm{Ar}}\right) ; 170.34\left(\mathrm{~d},{ }^{2} J_{\mathrm{PC}}=8.5 \mathrm{~Hz}, C=\mathrm{O}\right)$. IR (film) $\mathrm{v}_{(\mathrm{C}=\mathrm{O})} 1764, \mathrm{v}_{(\mathrm{P}=\mathrm{O})} 1264, \mathrm{v}_{(\mathrm{P}-\mathrm{O})} 1020$. Anal. Calcd. for $\mathrm{C}_{16} \mathrm{H}_{23} \mathrm{O}_{6} \mathrm{P}: \mathrm{C}$ 56.14, H 6.77. Found C, 56.23; H, 6.80.

\section{Acknowledgements}

The authors thank Professor Ryszard Bodalski (Technical University of Lodz) for stimulating discussions.

\section{References}

1. Gao, Y.; Sharpless, K. B. J. Am .Chem. Soc. 1988, 110, 7538.

2. Burgess, K.; Ho, K-K.; Ke, C.Y. J. Org. Chem. 1993, 58, 3767.

3. Burgess, K.; Ho, K-K. Tetrahedron Lett. 1992, 33, 5677.

4. Lim, D.; Burgess, K. J. Org. Chem. 1997, 62, 9382.

5. Hercouet, A.; Godbert, N.; Le Corre, M. Tetrahedron: Asymmetry 1998, 9, 2233.

6. Burgess, K.; Ho, K-K.; Moye-Sherman, D. Synlett 1994, 575.

7. Cativiela, C.; Díaz-de-Villegas, M. D. Tetrahedron: Asymmetry 2000, 11, 645.

8. De Simone, F.; Waser, J. Synthesis 2009, 3353.

9. Hercouet, A.; Le Corre, M.; Carboni, B. Tetrahedron Lett. 2000, 41, 197.

10. Choi, D.; Stables, J. P.; Kohn, M. Bioorg. Med. Chem. 1996, 4, 2105.

11. Page, P.; Blonski, C.; Perie, J. Bioorg. Med. Chem. 1999, 7, 1403.

12. Tsujigami, T.; Sugai, T.; Ohta, H. Tetrahedron: Asymmetry 2001, 12, 2543.

13. Paddon-Jones, G. C.; McErlean, C. S. P.; Hayes, P.; Moore, C. J.; Konig, W. A.; Kitching, W. J. Org. Chem. 2001, 66, 7487.

14. Allen, F. H., Acta Cryst. 1980, B36, 81.

15. Krawczyk, H.; Wąsek, K.; Kędzia, J; Wojciechowski, J; Wolf, W. M., Acta Cryst. 2008, C64, o24.

16. Meyer, E. A.; Castellano, R, K.; Diederich, F., Angew. Chem. Int. Ed. 2003, 42, 1210.

17. Bruker, SMART, Version 5.629, Bruker AXS, Madison, Wisconsin, 2003.

18. Bruker, SAINT-PLUS, Version 6.45A, Bruker AXS, Madison, Wisconsin, 2003. 
19. Bruker, $S A D A B S$ - Bruker Nonius area detector scaling and absorption correction, Version 2.10, (2006), Bruker AXS, Madison, Wisconsin, 2003.

20. Sheldrick, G. M. SHELXTL, Version 6.12; Bruker AXS, Madison, Wisconsin, 2003. 